\title{
Minimal inhibitory concentration distributions and epidemiological cutoff values of five antifungal agents against Sporothrix brasiliensis
}

\author{
Rodrigo Almeida-Paes ${ }^{1 /}$, Fábio Brito-Santos ${ }^{1}$, Maria Helena Galdino Figueiredo-Carvalho', \\ Ana Caroline Sá Machadoº , Manoel Marques Evangelista Oliveira', Sandro Antonio Pereira², \\ Maria Clara Gutierrez-Galhardo ${ }^{3}$, Rosely Maria Zancopé-Oliveira ${ }^{1}$
}

\begin{abstract}
${ }^{1}$ Fundação Oswaldo Cruz-Fiocruz, Instituto Nacional de Infectologia Evandro Chagas, Laboratório de Micologia, Rio de Janeiro, RJ, Brasil ${ }^{2}$ Fundação Oswaldo Cruz-Fiocruz, Instituto Nacional de Infectologia Evandro Chagas, Laboratório de Pesquisa Clínica em Dermatozoonoses em Animais Domésticos, Rio de Janeiro, RJ, Brasil

${ }^{3}$ Fundação Oswaldo Cruz-Fiocruz, Instituto Nacional de Infectologia Evandro Chagas, Laboratório de Pesquisa Clínica em Dermatologia Infecciosa, Rio de Janeiro, RJ, Brasil
\end{abstract}

BACKGROUND Sporothrix brasiliensis is the most virulent sporotrichosis agent. This species usually responds to antifungal drugs, but therapeutic failure can occur in some patients. Antifungal susceptibility tests have been performed on this species, but no clinical breakpoints (CBPs) are available. In this situation, minimal inhibitory concentration (MIC) distributions and epidemiological cutoff values (ECVs) support the detection of identification of resistant strains.

OBJECTIVES To study the MIC distributions of five antifungal drugs against $S$. brasiliensis and to propose tentative ECVs.

METHODS MICs of amphotericin B (AMB), itraconazole (ITR), ketoconazole (KET), posaconazole (POS), and terbinafine (TRB) against 335 S. brasiliensis strains were determined by the Clinical and Laboratory Standards Institute broth microdilution method.

FINDINGS The proposed ECV, in $\mu \mathrm{g} / \mathrm{mL}$, for AMB, ITR, KET, POS, and TRB were 4.0, 2.0, 1.0, 2.0, and 0.25, respectively. Percentages of wild-type strains in our population for the above antifungal drugs were 98.48, 95.22, 95.33, 100, and 97.67\%, respectively.

MAIN CONCLUSIONS These ECVs will be useful to detect strains with resistance, to define CBPs, and to elaborate specific therapeutic guidelines for $S$. brasiliensis. Rational use of antifungals is strongly recommended to avoid the emergence of resistant strains and ensure the therapeutic effectiveness of sporotrichosis.

Key words: antifungal drug - minimum inhibitory concentration - Sporothrix brasiliensis

Sporothrix brasiliensis is an emerging species of the Sporothrix complex, which is restricted to Brazil, and is highly pathogenic to humans and animals. This species is responsible for most sporotrichosis cases in this country, especially in the Southeast region, and is transmitted from infected cats to humans (Gutierrez-Galhardo et al. 2015). Studies using mouse models of experimental infection show that $S$. brasiliensis is highly virulent, resulting in high mortality rates in animals and a high fungal burden in several organs (Arrillaga-Moncrieff et al. 2009). In humans, this species is associated with some unusual manifestations of sporotrichosis, such as hypersensitivity reactions, disseminated infection in non-AIDS patients, central nervous system tropism, and ocular involvement (Almeida-Paes et al. 2014, Macedo et al. 2015).

Although this is a sympatric species (GutierrezGalhardo et al. 2015), sporotrichosis cases caused by $S$. brasiliensis can be found in other countries because of the

doi: 10.1590/0074-02760160527

Financial support: FAPERJ (grant no E-26/102.255/2013),

CNPq (grants no 305487/2015-9, 304976/2013-0),

PAPES/Fiocruz (Grants 407771/2013-3, 407693/2012-2).

+ Corresponding author: rodrigo.paes@ini.fiocruz.br

Received 7 December 2016

Accepted 16 January 2017 migration of humans, the transport of pet cats, or the return of international travellers from endemic areas. When these imported mycoses are diagnosed in non-endemic areas, the lack of experience of clinicians and laboratory workers can delay diagnosis and make clinical management and treatment more difficult (Lelievre et al. 2015).

The first drug choice for sporotrichosis treatment is itraconazole (ITR), with terbinafine (TRB) and potassium iodide as alternatives; amphotericin B (AMB) is indicated in cases of severe disease (Kauffman et al. 2007). Most patients infected with $S$. brasiliensis respond well to these drugs (Francesconi et al. 2011, Almeida-Paes et al. 2014, Macedo et al. 2015), although therapeutic failures have been reported (Almeida-Paes et al. 2016). In order to guide therapy, antifungal susceptibility tests were developed (CLSI 2008). For some fungus-antifungal drug combinations, there are clinical breakpoints (CBPs). CBPs categorise strains that are inhibited by drug concentrations usually achievable at the site of infection as susceptible and strains that are not inhibited by achievable concentrations of the agent administered under normal dosage schedules as resistant. These CBPs are based on the minimal inhibitory concentration (MIC) distributions for each drug and species, the biochemical, molecular, and pharmacodynamic properties of the antifungal drugs, and the clinical data related to these drugs with these MIC values (Alastruey-Izquierdo et al. 2015). Although a reference method has been described for 
Sporothrix schenckii MIC determinations, there are no defined CBPs for any species that causes sporotrichosis (CLSI 2008). When CBPs are unavailable, the establishment of epidemiological cutoff values (ECVs) provides a means to detect strains with emerging resistance against antifungal drugs. Strains with MIC values equal or less than the ECVs are defined as wild-type (WT) strains, i.e., strains that does not harbour any acquired resistance to a tested antifungal agent. In contrast, strains with MICs higher than the ECVs have elevated odds to present acquired or mutational resistance mechanisms to the tested drug (Espinel-Ingroff \& Turnidge 2016).

In the present study, a large collection of $S$. brasiliensis strains was tested against five different drugs to determine MIC distributions and to establish ECVs for each antifungal agent against this species.

\section{MATERIALS AND METHODS}

Strains - A total of 335 S. brasiliensis strains obtained from 2002 to 2015 were included in this study. These strains were obtained from individual cases of human sporotrichosis $(n=212)$, feline sporotrichosis $(n=122)$, and the environment $(\mathrm{n}=1)$. The Research Ethics Committee of Evandro Chagas National Institute of Infectious Diseases (INI)/Fiocruz approved this study under protocol number CAAE 26637014.8.0000.5262. The strains were identified as $S$. brasiliensis by partial sequencing of the calmodulin gene or T3B polymerase chain reaction (PCR) fingerprinting (Oliveira et al. 2012) and were stored at $-20^{\circ} \mathrm{C}$ until used. Strains were subcultured on potato dextrose agar (PDA) prior to antifungal testing.

Antifungal drugs - AMB, ITR, ketoconazole (KET), posaconazole (POS), and TRB were obtained from Sigma-Aldrich (St. Louis, MO). Two-fold serial dilutions of stock solutions of these antifungal agents prepared in dimethyl sulphoxide (DMSO) were performed to obtain working concentrations of antifungal drugs ranging from 0.015 to $8 \mathrm{mg} / \mathrm{L}$, in a constant concentration of $1 \%$ DMSO.

Antifungal susceptibility testing - Inocula of 1-5 $\times$ $10^{4}$ conidia/mL were prepared in sterile saline solution after incubation of each strain in PDA for seven days at $37^{\circ} \mathrm{C}$. The broth microdilution reference method was performed according the M38-A2 CLSI guidelines (CLSI 2008), using RPMI 1640 medium buffered to pH 7.0 with $0.165 \mathrm{~mol} / \mathrm{L}$ morpholinepropanesulfonic acid (Sigma-Aldrich). The different working concentrations of antifungal drugs were distributed into wells of round-bottom 96-well microplates. Wells containing RPMI-1640 medium with $1 \%$ DMSO and the fungal inoculum and without any antifungal agent were used as growth controls, or, when only medium with DMSO was added, as a sterility controls. Quality controls with the reference strains Aspergillus fumigatus ATCC 204305 and Aspergillus flavus ATCC 204304 were included in each microplate. Tests were validated only if MIC values for these strains were within the range described in the M38-A2 reference document. MICs were determined by visual inspection after $48-72 \mathrm{~h}$ of incubation at $35^{\circ} \mathrm{C}$, as described (CLSI 2008). In brief, for AMB, ITR, and POS, the MIC endpoints were the lowest concentrations that completely inhibited fungal growth. For KET, the MIC was the lowest concentration that resulted in a 50\% reduction in growth relative to that of the growth control, and, for TRB, it was the lowest concentration that resulted in at least an $80 \%$ reduction in growth relative to the control without the antifungal drug. All strains were tested at least twice against AMB, KET, ITR, and TRB. POS was tested only with strains isolated from human patients.

Analysis of results - GraphPad Prism 5 software was used for data analysis. The range, MIC50, MIC90, and geometric means with a $95 \%$ confidence interval (CI) were obtained by a descriptive statistics analysis. Frequency $\log _{2}$ MIC distributions of each antifungal drug were plotted as histograms (Turnidge et al. 2006). To compare the in vitro efficacy of azoles against $S$. brasiliensis, ITR, KET, VOR, and POS MICs were compared using the non-parametric Mann-Whitney test. This test was also used to compare the antifungal susceptibilities of the antifungal agents against strains of human or animal origin. A p $<0.05$ was considered statistically significant. ECVs were calculated as described previously (Espinel-Ingroff et al. 2014, Cafarchia et al. 2015), considering the modal MIC, the MIC distribution, the assumption that the WT population should cover three to five two-fold dilutions around the modal MIC, the assumption that an ECV should encompass at least $95 \%$ of WT organisms, and the inherent variability of the test, usually within one two-fold dilution. This variability can result from small lot-to-lot variations in media and reagents, the incubation atmosphere, imprecision in dilutions and inoculum preparation, and inaccuracies in endpoint visual determinations. Strains with MICs $\leq$ ECVs were classified as WT strains, and those with MICs > ECVs were classified as non-WT strains (Espinel-Ingroff \& Turnidge 2016). The probability of a strain with a MIC above the ECV exhibiting resistance to the drug was calculated using the probability mass function as previously described (Turnidge et al. 2006).

\section{RESULTS}

The MIC values of five antifungal drugs against $S$. brasiliensis are shown in Table I. In general, the MIC of TRB was at least two-fold lower than those observed for the other drugs, except for that the MIC of TRB was one-fold dilution lower than MIC of KET. Among the azoles, KET was the most effective antifungal drug ( $p<$ 0.0001 ), and the in vitro susceptibilities of $S$. brasiliensis to ITR and POS were similar to one another $(\mathrm{p}=0.7116)$. Trailing end points for these agents and $S$. brasiliensis were not encountered, as reported before for Aspergillus and other fungi (CLSI 2008).

Table II presents a comparison of MICs of AMB, KET, ITR, and TRB against strains isolated from human and cats with sporotrichosis. In general, KET and ITR were associated with similar susceptibilities in strains from these two sources, whereas the MICs of AMB and TRB were higher for strains of human origin.

The MIC distributions for AMB, ITR, KET, POS, and TRB against $S$. brasiliensis are presented in Figure. The modal MIC values (in $\mu \mathrm{g} / \mathrm{mL}$ ) at $72 \mathrm{~h}$ of incubation 
TABLE I

Minimal inhibitory concentrations (MIC) $(\mu \mathrm{g} / \mathrm{mL})$ of five antifungal drugs against Sporothrix brasiliensis

\begin{tabular}{lcccc}
\hline Antifungal drug & Range & GM $(95 \%$ CI $)$ & MIC50 & MIC90 \\
\hline Amphotericin B & $0.12-8.0$ & $1.097(1.014-1.188)$ & 1.0 & 4.0 \\
Itraconazole & $0.06-16.0$ & $0.762(0.695-0.835)$ & 1.0 & 2.0 \\
Ketoconazole & $0.03-2.0$ & $0.356(0.317-0.4)$ & 0.5 & 1.0 \\
Posaconazole & $0.06-2.0$ & $0.756(0.695-0.823)$ & 1.0 & 2.0 \\
Terbinafine & $0.015-1.0$ & $0.072(0.064-0.08)$ & 0.06 & 0.25 \\
\hline
\end{tabular}

CI: confidence interval; GM: geometric mean.

\section{TABLE II}

Comparison between minimal inhibitory concentrations (MIC) $(\mu \mathrm{g} / \mathrm{mL})$ of four antifungal agents against strains isolated from humans or cats with sporotrichosis

\begin{tabular}{|c|c|c|c|c|c|}
\hline \multirow[b]{2}{*}{ Antifungal } & \multicolumn{2}{|c|}{ Human strains } & \multicolumn{2}{|c|}{ Cat strains } & \multirow[b]{2}{*}{$\mathrm{p}$ value } \\
\hline & Median & GM $(95 \%$ CI $)$ & Median & GM $(95 \%$ CI $)$ & \\
\hline AMB & 1.0 & 1.249 (1.131-1.378) & 1.0 & $0.8822(0.7796-0.9984)$ & $<0.0001$ \\
\hline KET & 0.25 & $0.3407(0.2759-0.4207)$ & 0.5 & $0.3742(0.3282-0.4266)$ & 0.2786 \\
\hline ITR & 1.0 & $0.7798(0.6818-0.8919)$ & 1.0 & $0.7311(0.6655-0.8033)$ & 0.7750 \\
\hline TRB & 0.12 & $0.0943(0.0843-0.1055)$ & 0.03 & $0.0369(0.0317-0.0429)$ & $<0.0001$ \\
\hline
\end{tabular}

AMB: amphotericin B; CI: confidence interval; GM: geometric mean; ITR: itraconazole; KET: ketoconazole; TRB: terbinafine.

for these drugs were $1.0,1.0,0.5,1.0$, and 0.125 , respectively. The ECVs of $S$. brasiliensis were $4.0 \mu \mathrm{g} / \mathrm{mL}$ for AMB, $2.0 \mu \mathrm{g} / \mathrm{mL}$ for ITR, $1.0 \mu \mathrm{g} / \mathrm{mL}$ for KET, $2.0 \mu \mathrm{g} /$ $\mathrm{mL}$ for POS, and $0.25 \mu \mathrm{g} / \mathrm{mL}$ for TRB. All tested strains were classified as WT when treated with POS. Non-WT strains were detected, in increasing order, with AMB, TRB, KET, and ITR (Table III). These strains were isolated throughout the years 2003 to 2014, i.e., there was no temporal clustering of non-WT strains. The probability of non-WT strains exhibiting resistance against the tested drugs ranged from 95.01-99.94\%.

\section{DISCUSSION}

The MIC distributions for ITR, KET, POS, and TRB showed good susceptibility of $S$. brasiliensis to these drugs overall. The MIC distribution for AMB suggested moderate efficacy of this drug. Moreover, since the calculated ECV for this antifungal drug so high, it makes difficult to distinguish between wild type WT and non-wild WT type isolates. One possible weakness of this study was that MIC distributions were studied in strains isolated from a single centre. This limitation was mitigated by the fact that the isolates were obtained over seven years, different lots of broth microdilution reagents and drugs were used, and several qualified professionals read the MIC endpoints. This strategy was also used in a study performed in two centres to determine ECVs for unusual Candida species (Pfaller et al. 2011). Moreover, MIC ranges of other studies for $S$. brasiliensis antifungal susceptibility are within the MIC distributions observed in this study (Marimon et al. 2008, Ottonelli-Stopiglia et al. 2014, Rodrigues et al. 2014a, Borba-Santos et al. 2015, Almeida-Paes et al. 2016, Brilhante et al. 2016). It is also important to note that $S$. brasiliensis is currently found only in Brazil, and its major hyperendemic area is Rio de Janeiro state (Almeida-Paes et al. 2014, GutierrezGalhardo et al. 2015), where this study was performed.

CLSI reference method M38-A2 (CLSI 2008) is laborious and difficult to apply in most clinical laboratories, especially those with limited financial resources. One study showed very high agreement in S. schenckii ITR susceptibilities between the microdilution method and commercial tests such as Sensititre YeastOne and ATB Fungus (Alvarado-Ramirez \& Torres-Rodriguez 2007). Therefore, we recommend that laboratories unable to perform the microdilution assay use commercial kits to determine Sporothrix susceptibilities to the most common antifungal agents used in the treatment of sporotrichosis. Further studies to determine the ECVs of antifungal drugs in S. brasiliensis with commercial tests are needed.

The ECVs of $S$. brasiliensis calculated in this study were similar to the ECVs of Aspergillus spp. (EspinelIngroff \& Turnidge 2016), another filamentous genus described in the CLSI M38-A2 document (CLSI 2008). For fungi in the genus Aspergillus, ECVs are useful for determining whether an isolate harbours mutations in the Cyp51A gene (Espinel-Ingroff et al. 2010). Moreover, $\mathrm{ECV}$ are relevant in the use of POS in the treatment of an experimental mouse model of aspergillosis (Calvo et al. 2012). Despite the differences between these two organisms, the clinical pertinence of the Aspergillus ECV suggests that the ECVs proposed in this study for $S$. 

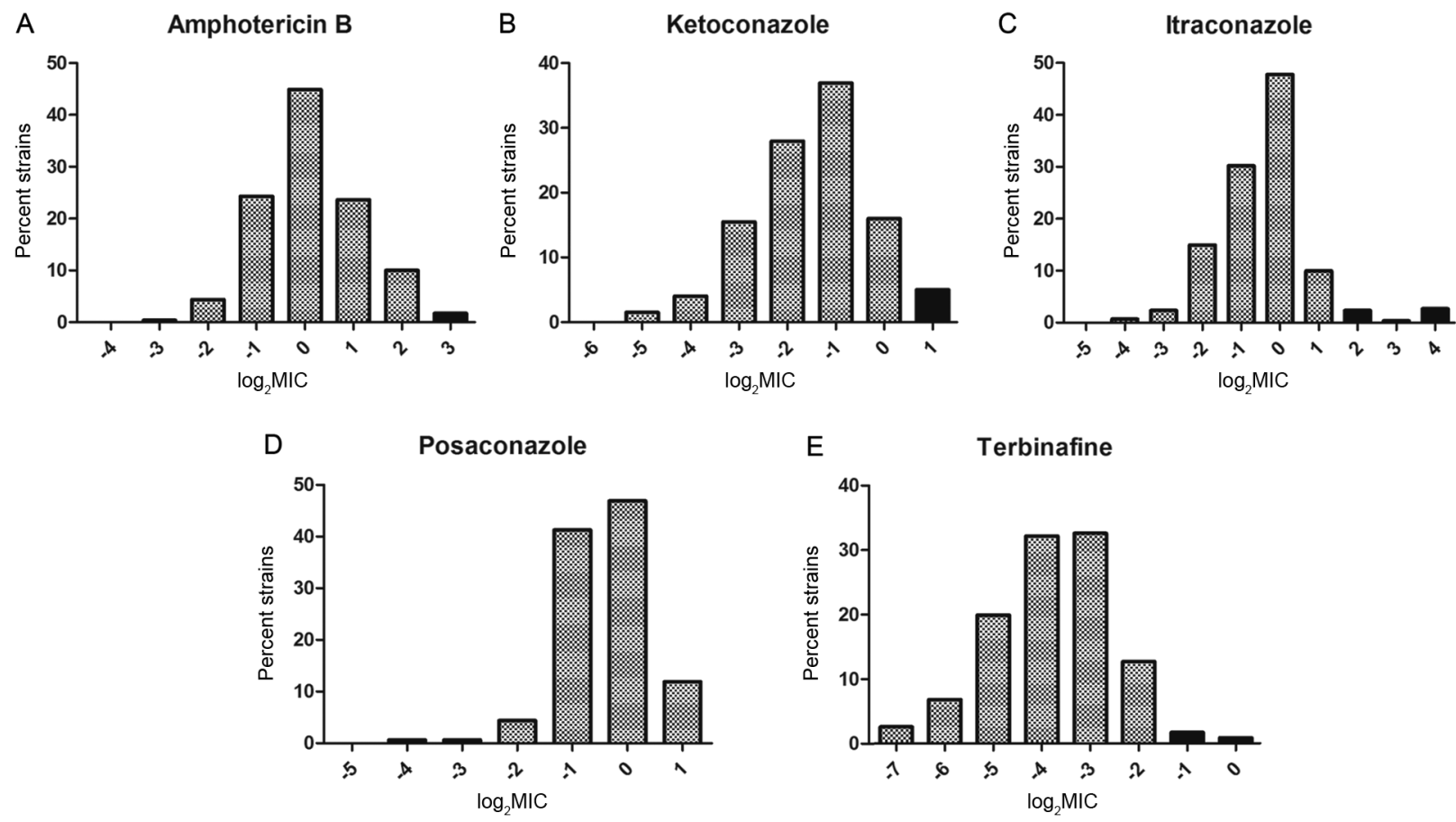

Minimal inhibitory concentration distribution of Sporothrix brasiliensis against amphotericin B (A), ketoconazole (B), itraconazole (C), posaconazole (D), and terbinafine (E), indicating wild-type strains (dotted bars) and non-wild-type strains (solid bars).

TABLE III

Proposed epidemiological cutoff values for five antifungal drugs against Sporothrix brasiliensis

\begin{tabular}{|c|c|c|c|c|}
\hline \multirow[b]{2}{*}{ Antifungal drug } & \multicolumn{2}{|c|}{$\mathrm{ECV}(\mu \mathrm{g} / \mathrm{mL})$} & \multirow[b]{2}{*}{ MIC (\%) of non-WT isolates } & \multirow[b]{2}{*}{ Probability to harbor resistance } \\
\hline & WT & Non-WT & & \\
\hline Amphotericin B & $\leq 4.0$ & $\geq 8.0$ & $8.0 \mu \mathrm{g} / \mathrm{mL}(1.52)$ & $99.05 \%$ \\
\hline Itraconazole & $\leq 2.0$ & $\geq 4.0$ & $\begin{array}{c}4.0 \mu \mathrm{g} / \mathrm{mL}(2.09) \\
8.0 \mu \mathrm{g} / \mathrm{mL}(0.30) \\
16.0 \mu \mathrm{g} / \mathrm{mL}(2.39)\end{array}$ & $\begin{array}{l}95.01 \% \\
99.24 \% \\
99.94 \%\end{array}$ \\
\hline Ketoconazole & $\leq 1.0$ & $\geq 2.0$ & $2.0 \mu \mathrm{g} / \mathrm{mL}(4.67)$ & $95.6 \%$ \\
\hline Posaconazole & $\leq 2.0$ & $\geq 4.0$ & - & - \\
\hline Terbinafine & $\leq 0.25$ & $\geq 0.5$ & $\begin{array}{l}0.5 \mu \mathrm{g} / \mathrm{mL}(1.55) \\
1.0 \mu \mathrm{g} / \mathrm{mL}(0.78)\end{array}$ & $\begin{array}{l}97.39 \% \\
99.68 \%\end{array}$ \\
\hline
\end{tabular}

ECV: epidemiological cutoff value; MIC: minimal inhibitory concentration; WT: wild-type.

brasiliensis could also may be useful for epidemiologic and clinical purposes.

Most patients with sporotrichosis in Rio de Janeiro, Brazil, where S. brasiliensis predominates (AlmeidaPaes et al. 2014, Gutierrez-Galhardo et al. 2015), have been treated with ITR and TRB (Francesconi et al. 2011, Almeida-Paes et al. 2016). To the best of our knowledge, POS has been used only once, to treat a single case of disseminated sporotrichosis that was refractory to AMB and ITR and that was caused by S. brasiliensis in a patient with AIDS, resulting in a clinical response and clearance of the fungus from the central nervous system (Paixão et al. 2015). However, this antifungal is seldom used because of its high cost and because the Brazilian government does not distribute this drug to treat mycotic infections. Oral KET has been described for the treatment of feline sporotrichosis, but it showed less effectiveness than oral ITR (Pereira et al. 2010). The similar susceptibilities to KET and ITR between cat and human strains in our study support the idea of clonal transmission of sporotrichosis between cats and humans in Rio de Janeiro (Rodrigues et al. 2014b). To the best of our knowledge, TRB was not used to treat domestic animals with sporotrichosis during the study period, and intralesional AMB was used only in association with oral ITR in cases refractory to this azole (Gremião et al. 2011). 
Significant differences in antifungal susceptibilities have been observed among pathogenic Sporothrix spp. In general, $S$. brasiliensis shows a better response to antifungal drugs, especially ITR (Marimon et al. 2008, Rodrigues et al. 2014a). Therefore, the ECVs described here should only be used in Sporothrix strains identified as $S$. brasiliensis by molecular methods (Oliveira et al. 2012, Rodrigues et al. 2014a).

A few strains with higher probability of exhibiting resistance to the antifungal drugs used in the treatment of sporotrichosis were found in our study. The indiscriminate use of these antifungal drugs to treat classical and non-aggressive forms of sporotrichosis and other mycoses may have led to the emergence of resistance mechanisms. Sporotrichosis can be treated with non-antimycotic drugs such as potassium iodide (Bonifaz et al. 2007, Macedo et al. 2015) and with non-pharmacologic strategies such as hyperthermia (Bonifaz et al. 2007) and cryosurgery (Almeida-Paes et al. 2016), or even with a combination of low drug dosages and hyperthermia (Haruna et al. 2006). The non-pharmacologic approaches are interesting because they often accelerate clinical cures, reducing treatment costs. Therefore, we strongly recommend the rational use of antimycotic drugs to delay the emergence of resistant strains and to preserve these therapies for more complex sporotrichosis cases.

The detection of strains with elevated MICs against AMB, KET, ITR, and TRB suggests the emergence of $S$. brasiliensis strains that are resistant to these drugs. The molecular characterisation of Sporothrix strains is of increasing interest worldwide, and related techniques will make it possible to easily diagnose $S$. brasiliensis infections in countries other than Brazil in the future. These ECVs may help clinicians to appropriately manage sporotrichosis caused by $S$. brasiliensis and to monitor emerging resistance against antifungal drugs used to treat this mycotic infection. Because MICs and ECVs can vary according to several factors, including incubation time or medium composition, it is extremely important that these ECVs be used with the same experimental conditions described here. Given that we were not able to obtain clinical data associated with all strains included in this study, a multicentric validation, corroborating data from experimental models of infection, and clinical correlations with Sporothrix ECVs are needed to devise better strategies for sporotrichosis treatment.

\section{AUTHORS' CONTRIBUTION}

Designed experiments - RA-P, SAP, MCG-G and RMZ-O; identified strains - MMEO; performed antifungal susceptibility tests - RA-P, FB-S, MHGF-C and ACSM; analysed data - RA-P, MMEO, SAP, MCG-G and RMZ-O; wrote the paper - RA-P, MCG-G and RMZ-O; revised and approved the final version of the paper - RA-P, FB-S, MHGF-C, ACSM, MMEO, SAP, MCG-G and RMZ-O.

\section{REFERENCES}

Alastruey-Izquierdo A, Melhem MSC, Bonfietti LX, RodriguezTudela JL. Susceptibility test for fungi: clinical and laboratorial correlations in medical mycology. Rev Inst Med Trop São Paulo. 2015; 57(Suppl. 19): 57-64.
Almeida-Paes R, Oliveira MME, Freitas DFS, do Valle ACF, Gutierrez-Galhardo MC, Zancopé-Oliveira RM. Refractory sporotrichosis due to Sporothrix brasiliensis in humans appears to be unrelated to in vivo resistance. Med Mycol. 2016; doi: 10.1093/ mmy/myw103.

Almeida-Paes R, Oliveira MME, Freitas DFS, Valle ACF, ZancopéOliveira RM, Gutierrez-Galhardo MC. Sporotrichosis in Rio de Janeiro, Brazil: Sporothrix brasiliensis is associated with atypical clinical presentations. PLoS Negl Trop Dis. 2014; 8(9): e3094.

Alvarado-Ramirez E, Torres-Rodriguez JM. In vitro susceptibility of Sporothrix schenckii to six antifungal agents determined using three different methods. Antimicrob Agents Chemother. 2007; 51(7): 2420-3.

Arrillaga-Moncrieff I, Capilla J, Mayayo E, Marimon R, Mariné M, Gené J, et al. Different virulence levels of the species of Sporothrix in a murine model. Clin Microbiol Infect. 2009; 15(7): 651-5.

Bonifaz A, Saúl A, Paredes-Solis V, Fierro L, Rosales A, Palacios C, et al. Sporotrichosis in childhood: clinical and therapeutic experience in 25 patients. Pediatr Dermatol. 2007; 24(4): 369-72.

Borba-Santos LP, Rodrigues AM, Gagini TB, Fernandes GF, Castro R, Camargo ZP, et al. Susceptibility of Sporothrix brasiliensis isolates to amphotericin B, azoles, and terbinafine. Med Mycol. 2015; 53(2): 178-88.

Brilhante RSN, Rodrigues AM, Sidrim JJC, Rocha MFG, Pereira SA, Gremião IDF, et al. In vitro susceptibility of antifungal drugs against Sporothrix brasiliensis recovered from cats with sporotrichosis in Brazil. Med Mycol. 2016; 54(3): 275-9.

Cafarchia C, Iatta R, Immediato D, Puttilli MR, Otranto D. Azole susceptibility of Malassezia pachydermatis and Malassezia furfur and tentative epidemiological cut-off values. Med Mycol. 2015; 53(7): 743-8.

Calvo E, Pastor FJ, Sutton DA, Fothergill AW, Rinaldi MG, Salas V, et al. Are epidemiologic cut-off values predictors of the in vivo efficacy of azoles in experimental aspergillosis? Diagn Microbiol Infect Dis. 2012; 74(2): 158-65.

CLSI - Clinical and Laboratory Standards Institute. Reference method for broth dilution antifungal susceptibility testing of filamentous fungi. 2nd ed. Wayne: Clinical and Laboratory Standards Institute; 2008. $52 \mathrm{pp}$.

Espinel-Ingroff A, Diekema DJ, Fothergill A, Johnson E, Pelaez T, Pfaller MA, et al. Wild-type MIC distributions and epidemiological cutoff values for the triazoles and six Aspergillus spp. for the CLSI broth microdilution method (M38-A2 Document). J Clin Microbiol. 2010; 48(9): 3251-7.

Espinel-Ingroff A, Pfaller MA, Bustamante B, Canton E, Fothergill A, Fuller J, et al. Multilaboratory study of epidemiological cutoff values for detection of resistance in eight Candida species to fluconazole, posaconazole, and voriconazole. Antimicrob Agents Chemother. 2014; 58(4): 2006-12.

Espinel-Ingroff A, Turnidge J. The role of epidemiological cutoff values (ECVs/ECOFFs) in antifungal susceptibility testing and interpretation for uncommon yeasts and moulds. Rev Iberoam Micol. 2016; 33(2): 63-75.

Francesconi G, Valle ACF, Passos SRL, Barros MBL, Almeida-Paes $\mathrm{R}$, Curi ALL, et al. Comparative study of $250 \mathrm{mg} /$ day terbinafine and $100 \mathrm{mg} /$ day itraconazole for the treatment of cutaneous sporotrichosis. Mycopathologia. 2011; 171(5): 349-54.

Gremião IDF, Schubach TMP, Pereira SA, Rodrigues AM, Honse CO, Barros MBL. Treatment of refractory feline sporotrichosis with a combination of intralesional amphotericin B and oral itraconazole. Aust Vet J. 2011; 89(9): 346-51. 
Gutierrez-Galhardo MC, Freitas DFS, Valle ACF, Almeida-Paes R, Oliveira MME, Zancopé-Oliveira RM. Epidemiological aspects of sporotrichosis epidemic in Brazil. Curr Fungal Infect Rep. 2015; 9(4): 238-45.

Haruna K, Shiraki Y, Hiruma M, Ikeda S, Kawasaki M. A case of lymphangitic sporotrichosis occurring on both forearms with a published work review of cases of bilateral sporotrichosis in Japan. J Dermatol. 2006; 33(5): 364-7.

Kauffman CA, Bustamante B, Chapman SW, Pappas PG. Clinical practice guidelines for the management of sporotrichosis: 2007 update by the Infectious Diseases Society of America. Clin Infect Dis. 2007; 45(10): 1255-65.

Lelievre L, Borderie V, Garcia-Hermoso D, Brignier AC, Sterkers M, Chaumeil C, et al. Imported Pythium insidiosum keratitis after a swim in Thailand by a contact lens-wearing traveler. Am J Trop Med Hyg. 2015; 92(2): 270-3.

Macedo PM, Lopes-Bezerra LM, Bernardes-Engemann AR, Orofino-Costa R. New posology of potassium iodide for the treatment of cutaneous sporotrichosis: study of efficacy and safety in 102 patients. J Eur Acad Dermatol Venereol. 2015; 29(4): 719-24.

Marimon R, Serena C, Gené J, Cano J, Guarro J. In vitro antifungal susceptibilities of five species of Sporothrix. Antimicrob Agents Chemother. 2008; 52(2): 732-4.

Oliveira MME, Sampaio P, Almeida-Paes R, Pais C, GutierrezGalhardo MC, Zancope-Oliveira RM. Rapid identification of Sporothrix species by T3B fingerprinting. J Clin Microbiol. 2012; 50(6): 2159-62.
Ottonelli-Stopiglia CD, Magagnin CM, Castrillón MR, Mendes SDC, Heidrich D, Valente P, et al. Antifungal susceptibilities and identification of species of the Sporothrix schenckii complex isolated in Brazil. Med Mycol. 2014; 52(1): 56-64.

Paixão AG, Gutierrez-Galhardo MC, Almeida-Paes R, Nunes EP, Gonçalves MLC, Chequer GL, et al. The difficult management of disseminated Sporothrix brasiliensis in a patient with advanced AIDS. AIDS Res Ther. 2015; 12: 16.

Pereira SA, Passos SRL, Silva JN, Gremião IDF, Figueiredo FB, Teixeira $\mathrm{JL}$, et al. Response to azolic antifungal agents for treating feline sporotrichosis. Vet Rec. 2010; 166(10): 290-4.

Pfaller MA, Castanheira M, Diekema DJ, Messer SA, Jones RN. Triazole and echinocandin MIC distributions with epidemiological cutoff values for differentiation of wild-type strains from nonwild-type strains of six uncommon species of Candida. J Clin Microbiol. 2011; 49(11): 3800-4.

Rodrigues AM, de Hoog GS, Pires DC, Brilhante RSN, Sidrim JJC, Gadelha MF, et al. Genetic diversity and antifungal susceptibility profiles in causative agents of sporotrichosis. BMC Infect Dis. 2014a; 14: 219.

Rodrigues AM, de Hoog GS, Zhang Y, Camargo ZP. Emerging sporotrichosis is driven by clonal and recombinant Sporothrix species. Emerg Microbes Infect. 2014b; 3(5): e32.

Turnidge J, Kahlmeter G, Kronvall G. Statistical characterisation of bacterial wild-type MIC value distributions and the determination of epidemiological cut-off values. Clin Microbiol Infect. 2006; 12(5): 418-25. 